1 Targeted gene integration using the combination of a sequence-specific DNA-binding protein

\title{
2 and phic31 integrase
}

3

4 Hideyuki Nakanishi, ${ }^{1,2}$ Yuriko Higuchi, ${ }^{3,4}$ Fumiyoshi Yamashita, ${ }^{1}$ and Mitsuru Hashida ${ }^{1,5}$

$5 \quad{ }^{1}$ Department of Drug Delivery Research, Graduate School of Pharmaceutical Sciences, Kyoto

6 University, 46-29 Yoshida Shimoadachi-cho, Sakyo-ku, Kyoto 606-8501, Japan

$7 \quad{ }^{2}$ Japan Society for the Promotion of Science (JSPS), Sumitomo-Ichibancho FS Bldg., 8 Ichibancho,

8 Chiyoda-ku, Tokyo 102-8472, Japan

$9{ }^{3}$ Institute for Innovative NanoBio Drug Discovery and Development, Graduate School of 10 Pharmaceutical Sciences, Kyoto University, 46-29 Yoshida Shimoadachi-cho, Sakyo-ku, Kyoto 606-

118501 , Japan

$12{ }^{4}$ PRESTO, Japan Science and Technology Agency (JST), Kawaguchi Center Building 4-1-8, Honcho, 13 Kawaguchi-shi, Saitama 332-0012, Japan

$14{ }^{5}$ Institute for Integrated Cell-Material Sciences (iCeMS), Kyoto University, 69 Yoshida Ushinomiya15 cho, Sakyo-ku, Kyoto 606-8501, Japan

16 Correspondence should be addressed to Mitsuru Hashida (hashidam@pharm.kyoto-u.ac.jp)

17 This work was done in Kyoto, Japan.

18 Corresponding author's address: Department of Drug Delivery Research, Graduate School of 19 Pharmaceutical Sciences, Kyoto University, 46-29 Yoshida Shimoadachi-cho, Sakyo-ku, Kyoto 60620 8501, Japan

21 Telephone: $+81-75-753-4545$ Fax: +81-75-753-4575 Email: hashidam@pharm.kyoto-u.ac.jp

(C) 2014. This manuscript version is made available under the Elsevier user license http://www.elsevier.com/open-access/userlicense/1.0/ 


\section{Abstract}

2

3

5

6
PhiC31 integrase-based vectors can integrate therapeutic genes selectively into attP or pseudo-attP sites in genomes, but considerable numbers of pseudo-attP sites in human genomes exist inside endogenous gene-coding regions. To avoid endogenous gene disruptions, we aimed to enhance the integration site-specificity of the phiC31 integrase-based vector using a sequence-specific DNAbinding protein containing Gal4 and LexA DNA-binding motifs. The dual DNA-binding protein was designed to tether the UAS-containing donor vector to the target sequence, the LexA operator, and restrict integration to sites close to the LexA operator. To analyze the site-specificity in chromosomal integration, a human cell line having LexA operators on the genome was established, and the cell line was transfected with donor vectors expressing the DNA-binding protein and the phiC31 integrase expression vector (helper vector). Quantitative PCR indicated that integration around the LexA operator was 26-fold higher with the UAS-containing donor vector than with the control. Sequence analysis confirmed that the integration occurred around the LexA operator. The dual DNA-binding protein-based targeted integration strategy developed herein would allow safer and more reliable genetic manipulations for various applications, including gene and cell therapies.

\section{Keywords}

Genomic integration; Site-specific integration; Integrase; DNA binding protein; Gene therapy 


\section{Introduction}

Chromosomal integration enables sustained transgene expression and cell divisiondependent replication of transgenes. Therefore, integrative vectors have often been applied to gene therapies (Calos, 2006), reprogramming (Takahashi and Yamanaka, 2006), induction of differentiation (Lacoste et al., 2009), tumor-model establishments (Carlson et al., 2005) and production of recombinant proteins (Tomita et al., 2003). PhiC31 integrase, a serine recombinase of Streptomyces phage, is widely used for chromosomal integration (Calos, 2006). In its natural context, phiC31 integrase integrates phage genomes into bacterial genomes by recombination between phage attP sites and bacterial attB ones (Thorpe and Smith, 1998). PhiC31 integrase can also react with pseudo-attP sequences that partially match with bacterial attP sequences, and thereby integrate plasmid vectors containing attB sequences (called "donor vectors") into the genomes of many organisms, including yeasts (Thomason et al., 2001), insects (Groth et al., 2004), amphibians (Allen and Weeks, 2005), birds (Leghton et al., 2008), and mammals, in both cultured cells (Groth et al., 2000 and Thyagarajan et al., 2001) and adult animals (Olivares et al., 2002). Such attP or pseudo-attP-targeting characteristics of phiC31 integrase-based vector systems enable site-specific integration, unlike other integrative vectors including lentiviral vectors or Sleeping Beauty (Vink et al., 2009), piggyBac (Nakanishi et al., 2010 and 2011), and Tol2 (Grabundzija et al., 2010) transposon-based vectors. Gene integration into or close to endogenous genes can disrupt or dysregulate their function, and sometimes induces severe adverse effects such as cancer (Hacein-Bey-Abina et al., 2003). In addition, random integration of sites results in a variegation of integrated transgene expression, which is called "position effect variegation" (Robertson et al., 1995). While some newer techniques, including transcription activator-like effector nucleases (TALENs) (Cermak et al., 2011 and Miller et al., 2011) and clustered regularly interspaced short palindromic repeats (CRISPR)/Cas9 (Cong et al., 2013 and Mali et al., 2013), are available for site-specific chromosomal integration, a phiC31 integrase-based vector system still remains useful because it allows in vivo gene integration in adult mammals (Olivares et al., 2002). However, phiC31 integrase-based vector systems are not sufficient in terms of site-specificity of integration, considering 
that the human genome has 202-764 pseudo-attP sequences, about $38.7 \%$ of which are associated with endogenous genes (Chalberg et al., 2006).

In addition to the development of mutant integrases that have higher integration sitespecificity (Gersbach et al., 2010 and Keravala et al., 2009), utilization of sequence-specific DNAbinding proteins might be a promising approach for enhancing the integration site-specificity of phiC31 integrase-based vectors. It could be more easily adapted to an arbitrary target sequence by customizing sequence-specific DNA-binding motifs such as zinc finger (Carroll et al., 2006, Mandell and Barbas 2006, Umov et al., 2005, and Wright et al., 2006) or transcription activator-like effector (TALE) (Cermak et al., 2011, Miller et al., 2011, and Zhang et al., 2011). Enhanced site-specificity of integration due to fusion with DNA-binding proteins has been achieved with transposase (Ammar et al., 2012, Ivics et al., 2007, Kettlun et al., 2011, Lacoste et al., 2009, Maragathavally et al., 2006, Owens et al., 2012, Voigt et al., 2012, and Yant et al., 2007), retroviral integrase (Tan et al., 2004 and 2006), Tn3 resolvase and Gin invertase (Gordley et al., 2009). However, it has also been suggested that the coupling of phiC31 integrase with the DNA-binding protein results in loss of activity (Shinohara et al., 2007). On the other hand, Ivics et al. proposed a conceptually different approach whereby a dual DNA-binding protein is utilized to tether the donor vector to the binding target in chromosomes and restricts the integration to nearby sites (Ivics et al., 2007). They demonstrated that co-transfection of cells with dual LexA/SAF-box (or LexA/TetR) DNA-binding proteins successfully enhanced the site-specificity of Sleeping Beauty transposase-mediated integration. We thought that this technique might be applicable to the phiC31 integrase-based vector system, since phiC31 integrase itself is in a native form.

In this context, the present study aimed to further improve the site-specificity of a phiC31 integrase-based vector system by combination with a dual DNA-binding protein. We made some modifications in the design of the donor vector as compared to the methods of Ivics et al. We developed a donor vector which carries both an attP sequence and the expression cassettes of a dual DNA-binding protein, so that we could ensure the expression of the DNA-binding protein and minimize the risk of nonspecific integration. In addition, to decrease the probability that phiC31 integrase-mediated 
1 integration precedes the DNA-binding protein-mediated tethering, we transfected cells with the helper

2 vector 1 or 2 days later than the donor vectors. These modifications allowed us to successfully increase

3 the percentage of the targeted integration by 4.5 -fold in the inter-plasmid integration assay and up to

426 -fold in the chromosomal integration assay. The present study is the first demonstration that

5 sequence-specific DNA-binding proteins can limit the chromosomal integration due to phiC31

6 integrase-based vectors to the more specific sites.

7

Materials and Methods

9

pDNA

KOD-plus ver.2 or KOD-plus Neo (Toyobo, Osaka, Japan) was used for PCRs to prepare inserts, and Rapid DNA Dephos \& Ligation Kit (Roche Diagnostics, Tokyo, Japan) or Mighty Cloning Kit (blunt end) (Takara Bio, Otsu, Japan) was used for ligations. All pDNAs were amplified in the $E$. coli strains DH5 $\alpha$ or HST08, isolated and purified using PureYield plasmid Miniprep Kit (Promega, Tokyo, Japan). For details of pDNA construction, see supplementary methods.

\section{Cell culture}

HEK293 and Hela cells were maintained in Dulbecco's modified Eagle's essential medium containing $10 \%$ fetal bovine serum.

\section{Establishment of a Hela-attPlex4R stable cell line}

Hela cells were transfected with pIR-attPlex4R and pFerH-PBTP using XtremeGene9

(Roche Diagnostics). The transfected cells were selected by antibiotic G418 (Nacalai Tesque, Kyoto,

24 Japan) over 2 weeks from day 2 onward, and cloned. To calculate the pIR-attPlex4R-derived transposons/endogenous RNaseP gene copy number, real-time PCR was performed with genomic DNA extracted from clone cells and digested with restriction enzymes BssHII and HindIII, using a

27 Light-Cycler instrument (Roche Diagnostics) and SYBR Premix Ex Taq (Takara Bio). The sequences 
1 of the primer sets used to determine the copy numbers of the neomycin-resistance gene in the

2 transposon and endogenous RNaseP gene were CGGATGGAAGCCGGTCTTGTC +

3 AgAAGGCGATAGAAGGCGATG and AGATTTGGACCTGCGAGCG +

4 GAGCGGCTGTCTCCACAAGT, respectively. pVITRO1-neo-RNasePfragment digested with

5 BssHII and HindIII that contained both the neomycin-resistance gene and a fragment of the RNaseP

6 gene was used to generate a standard curve.

7

8 Assay of Renilla luciferase activity

9 HEK293 cells were seeded onto 6- or 12-well plates. Eighteen hours later, the cells were

10 transfected with the indicated amount of pDNA using XtremeGene9 and then lysed using lysis buffer

$11(0.05 \%$ Triton X-100, 2 mM EDTA, $0.1 \mathrm{M}$ Tris, $\mathrm{pH} 7.8)$ at the indicated times. The Renilla luciferase

12 activity of the cell lysate was measured using a Biolux Gaussia luciferase assay kit (New England

13 BioLabs Japan, Tokyo, Japan) and Lumat LB 9507 (EG \& G Berthold, Bad Wildbad, Germany).

\section{Colony-counting assay}

Hela cells $\left(2.5 \times 10^{4}\right.$ cells $)$ were seeded onto $24-w e l l$ plates, and $18 \mathrm{hr}$ later transfected with the indicated amount of DNA using XtremeGene9. Two days after transfection, the cells were harvested, and $10 \%$ or $90 \%$ of the cells were transferred to 6 -well plates and maintained in medium containing $3 \mu \mathrm{g} / \mathrm{ml}$ blasticidin S (Invivogen) for two weeks. To count blasticidin-resistant colonies, cells were fixed with 4\% paraformaldehyde in PBS (Wako Pure Chemical Industries, Osaka, Japan)

21 for 10 min and stained with $0.2 \%$ methylene blue (Wako Pure Chemical Industries) in PBS. The numbers of colonies were corrected by the dilution ratio.

\section{Analysis of integration site-specificity in inter-plasmid integration}


1 kit (Sigma-Aldrich Japan, Tokyo, Japan). The ligation products were used to transform the E. coli

2 strain DH5 $\alpha$ or HST08. pDNA was purified from E. coli resistant to both kanamycin and blasticidin

3 using a PureYield plasmid Miniprep Kit (Promega). Purified pDNA was digested with restriction

4 enzymes either BamHI, NdeI, SpeI, BstZ17I plus NdeI. These digested pDNAs were electrophoresed

5 on $1 \%$ agarose $\mathrm{S}$ (Nippon Gene, Tokyo, Japan) gel to determine which recipient vectors were

6 integrated with donor vectors.

8 Analysis of targeted chromosomal integration

Hela-attPlex $4 \mathrm{R}$ cells $\left(5 \times 10^{4}\right.$ cells $)$ were seeded onto 6 -well plates, and $18 \mathrm{hr}$ later transfected

10 with the indicated amount of the donor vectors using XtremeGene9. One or two days after transfection

11 of the donor vectors, the cells were transfected with the indicated amount of pCMV-int. From three

12 days after transfection of the donor vectors, the cells were maintained in medium containing $3 \mu \mathrm{g} / \mathrm{ml}$

13 blasticidin S for 18 days. After blasticidin selection, DNA was extracted from these cells using a

14 Genelute mammalian genomic DNA extraction kit. For quantitative analysis of the attR and

15 blasticidin-resistance gene copy numbers, real-time PCR was performed with extracted DNA using a

16 Light-Cycler instrument (Roche Diagnostics) and SYBR Premix Ex Taq (Takara Bio). The sequences

17 of the primer sets used to determine the copy numbers of the att $R$ and blasticidin-resistance gene were tcgagGCATCAAGCTAATTC + AGTACGCCCCCTATTGACG and gaagacettcaacatctctcage + 19 atcttctcagtggegacctc, respectively. A targeted integration product containing both the attR and blasticidin-resistance genes was obtained by the plasmid rescue method, and used to generate a 21 standard curve.

\section{Analysis of integration sites by plasmid rescue}

To analyze phiC31 integrase-mediated integration sites, Hela-attPlex4R cells $\left(5 \times 10^{4}\right.$ cells $)$ were transfected with pTargetB2(4x)-LexA-Rluc. Two days later, the cells were transfected with pCMV-int. XtremeGene9 was used for both transfections. From the next day, cells were cultured in medium containing $3 \mu \mathrm{g} / \mathrm{ml}$ blasticidin $\mathrm{S}$ for 18 days. To analyze piggyBac transposon integration 
1 sites, HEK293 cells $\left(1.6 \times 10^{6}\right.$ cells $)$ were transfected with $2.0 \mu \mathrm{g}$ pTarget-NLS-Rluc or pTarget-LexA-

2 Rluc and $2.0 \mu \mathrm{g}$ pFerH-PBTP. Two days later, the cells were harvested and transferred to $100 \mathrm{~mm}$

3 plates and propagated in medium containing $3 \mu \mathrm{g} / \mathrm{ml}$ blasticidin S. DNA was isolated from these cells

4 using a Genelute mammalian genomic DNA extraction kit, and digested using restriction enzyme NheI,

5 SpeI, and XbaI for the Hela-attPlex4R genome and BglII and BamHI for the HEK293 genome. After

6 digestion by these restriction enzymes, the DNA was purified using a Genelute PCR Clean-up Kit

7 (Sigma-Aldrich Japan) and ligated using a Rapid DNA Dephos \& Ligation Kit (Roche Diagnostics)

8 or Ligation convenience kit (Nippon Gene). The ligation products were used to transform E. coli Strain

9 DH5 $\alpha$ or HST08. pDNA was purified from blasticidin-resistant E coli using a PureYield plasmid

10 Miniprep Kit. The nucleotide sequences of the pDNA were sequenced using a BigDye Terminator v3.1

11 Cycle Sequencing Kit (Life Technologies Japan, Tokyo, Japan) and ABI3100xl (Life Technologies

12 Japan). UC Santa Cruz BLAT was used to map integration sites.

\section{Results}

\section{Vector design to analyze inter-plasmid integration}

The phiC31 integrase-based vector system consists of two vector types. One is a donor vector that contains the $a t t B$ sequence, and the other is a helper vector that expresses phiC31 integrase. PhiC31 integrase expressed by helper vectors integrates donor vectors into chromosomes.

To enhance the site-specificity of phiC31 integrase-mediated integration, we designed the expression cassette of a dual DNA-binding protein which tethers the donor vector at the target sequence. The dual DNA-binding protein contains a Gal4 DNA-binding domain and LexA DNAbinding domain, which recognize an upstream activation sequence (UAS) and a LexA operator 24 sequence, respectively. Here, the LexA operator sequence was assumed to be a model of binding targets in chromosomes. Tethering of the donor vector by the dual DNA-binding protein limits phiC31 integrase-mediated integration to the attP sites close to its target sequence (Fig. 1). pTargetB-LexA- 
1 protein and 4 copies of UAS were inserted in addition to the Renilla luciferase gene. The donor vector

2 named pTargetB-NLS-Rluc was also constructed as a negative control. pTargetB-NLS-Rluc was the

3 same as pTargetB-LexA-Rluc, except that it expressed a DNA-binding protein lacking the LexA DNA-

4 binding domain. pAttB-UAS-Rluc was another negative control donor vector, which had no

5 expression cassette of DNA-binding protein. On the other hand, pCMV-int and pSV40-int were

6 constructed as helper vectors that express phiC31 integrase. To investigate whether the integration of

7 pTargetB-LexA-Rluc is dependent on the distance between the target sequence for the DNA-binding

8 protein and attP sequence, eight recipient vectors (i.e., pAttP-lex1 4F and 1 4R) having different

$9 \quad a t t P$-sequence orientations and different distances between the LexA operator and attP sequences were

10 constructed (Fig. 2).

11

Effect of the DNA-binding protein on the integration efficiency

It was preliminarily investigated whether binding of the DNA-binding protein to the donor

vector prevents phiC31 integrase from interacting with the donor vector. Following transfection of HEK293 cells with pTargetB-NLS-Rluc or pAttB-UAS-Rluc and with or without pCMV-int, sustainability of the gene expression of Rluc was evaluated as an indicator of integrations. When the effect of co-transfection with pCMV-int on the Rluc expression was evaluated on day 12, the sustained gene expression was enhanced by 2.32 -fold and 1.45 -fold in pTargetB-NLS-Rluc-transfected and pAttB-UAS-Rluc-transfected cells, respectively (Fig. 3). It should be remembered that the DNAbinding protein could be expressed and associated with the donor vector in pTargetB-NLS-Rluc but this was not the case in pAttB-UAS-Rluc. Nevertheless, the enhancement of sustained gene expression by phiC31 integrase was not lower in pTargetB-NLS-Rluc. This suggests that phiC31 integrase-mediated integration was not inhibited even though the DNA-binding protein bound to the donor vectors.

\section{Integration site-specificity in inter-plasmid integration}


1 in an inter-plasmid integration assay. To avoid phiC31 integrase-mediated inter-plasmid integration

2 during the amplification process in E. coli (Thorpe and Smith, 1998), pSV40-int was used as a helper

3 vector instead of pCMV-int (Fig. 2). pSV40-int was chosen because, unlike the CMV promoter, the

4 SV40 promoter does not express proteins in E. coli (Goussard et al., 2003). A preliminary experiment

5 indicated that pSV40-int cannot integrate donor vectors in E. coli but can do so in Hela cells (data not 6 shown).

First, Hela cells were transfected simultaneously with pSV40-int as a helper vector,

8 pTargeB-NLS-Rluc or pTargetB-LexA-Rluc as donor vectors, and pAttP-lex1-4F and 1-4R as

9 recipient vectors. Two days later, DNA was extracted from these cells and transformed to E. coli.

10 Taking into account that the inter-plasmid integration products contain blasticidin- and kanamycin-

11 resistance genes originated from donor and recipient vectors, respectively, transformed E. coli was

12 selected by both blasticidin and kanamycin. The inter-plasmid integration products extracted from $E$.

13 coli were digested with restriction enzymes and subjected to gel electrophoresis to determine to what

14 extent each recipient vector was integrated. Figure $4 \mathbf{a}$ and $\mathbf{b}$ shows the percentages of integration into

15 each recipient vector per total integration, and the ratio of the pTargetB-LexA-Rluc transfected group

16 per the pTargetB-NLS-Rluc group, respectively. pTargetB-LexA-Rluc was designed to express a

17 DNA-binding protein (i.e., the Gal4-LexA DNA-binding protein) that could bind to both the donor

18 and recipient vectors, whereas pTargetB-NLS-Rluc expressed a protein that bound only to the donor

19 vector. As shown in Fig. $\mathbf{4 b}$, the percentage of integration into pAttP-lex4R, which has the smallest

20 LexA-attP distance of $219 \mathrm{bp}$, was increased 4.5-fold in the pTargetB-LexA-Rluc group.

\section{Cell line establishment and vector construction for the analysis of chromosomal integration}

Prior to the evaluation of Gal4-LexA DNA-binding protein-enhanced integration sitespecificity in chromosomes, we established a stable cell line designated Hela-attPlex4R, in which both the attP and LexA operator sequences were chromosomally integrated. pAttP-lex4R was selected for

26 the integration to establish Hela-attPlex4R, based on the results of the inter-plasmid integration assay

27 (Fig. 4). At first, pAttP-lex4R was linearized and inserted into piggyBac transposon (hereafter pIR- 
1 attPlex4R). Then, pIR-attPlex4R together with the piggyBac transposase expression vector pFerH-

2 PBTP were transfected into Hela cells to integrate the transposon containing the pAttP-lex4R-derived

3 sequence into chromosomes (Fig. 5). Following selection, a stably integrated HeLa-attPlex4R cell

4 clone was obtained and subjected to quantitative evaluation of chromosomal integration. Integration

5 of attPlex4R sequences into the genome was evaluated with primers for neomycin-resistance gene in

6 the transposon, in reference with endogenous RNaseP gene. Real-time PCR analysis following

7 extraction and digestion of genomic DNA revealed that the number of integrated copies was 15 copies

8 per haploid genome in the Hela-attPlex4R clone.

9 The Hela-attPlex4R thus established was transfected with pTargetB-NLS-Rluc or pTargetB-

10 LexA-Rluc with pCMV-int. Unfortunately, transfection with these vectors produced very few

11 blasticidin-resistant cells (data not shown), despite the fact that the donor vectors contained the

12 blasticidin-resistance gene. We redesigned the donor vector constructs so that they contained the attB

13 sequence at a different position (Fig. 6a), taking into account a previous report that the location of the

$14 a t t B$ sequences in the donor vectors affects sustained gene expression by the phiC31 integrase-based

15 vector system (Watanabe et al., 2011). To minimize the effect on blasticidin-resistance gene expression,

16 the $a t t B$ sequence was placed apart from the blasticidin-resistance gene in the newly developed donor

17 vector construct pTargetB2-NLS-Rluc (Fig. 6a). As shown in Fig. 6b and c, transfection with

18 pTargetB2-NLS-Rluc and pCMV-int provided significantly more blasticidin-resistant colonies than

19 that with pTargetB-NLS-Rluc and pCMV-int.

20 Considering that displacement of the attB sequence was effective for increased expression

21 of the blasticidin-resistance gene, new donor vectors that expressed the Gal4-LexA DNA-binding

22 protein were constructed and designated pTargetB2(0x)-LexA-Rluc, pTargetB2(4x)-LexA-Rluc,

23 pTargetB2(18x)-LexA-Rluc, and pTargetB2(66x)-LexA-Rluc according to the number of UAS repeats

24 (Fig. 6a). We expected that binding between the donor vectors and the Gal4-LexA DNA-binding

25 protein would become more likely as the number of UAS increases.

\section{Integration site-specificity in chromosomal integration}


2 were transfected with pCMV-int and either pTargetB2-NLS-Rluc, pTargetB2(0x)-LexA-Rluc, 3 pTargetB2(4x)-LexA-Rluc, pTargetB2(18x)-LexA-Rluc, or pTargetB2(66x)-LexA-Rluc. To allow the 4 DNA-binding protein to be expressed and tether the donor vectors in advance, the cells were 5 transfected with the donor vectors 1 or 2 days prior to transfection with pCMV-int. After transfections 6 of pCMV-int, the cells were subjected to blasticidin selection and extraction of genomic DNA. 7 Quantitative PCR of genomic DNA was performed using forward and reverse primers designed to 8 anneal to a sequence neighboring the LexA operator and a sequence inside a donor vector, respectively.

9 These primers allowed us to count the copies of the donor vector integrated into the attP close to the 10 LexA operator sequence (Fig. 7a). The copy number of total donor vectors was also evaluated as an 11 internal control. As for the 1-day delay of pCMV-int transfection, the targeted integration efficiency 12 of pTargetB2(4 18x)-LexA-Rluc was minimally higher than that of the negative controls (pTargetB213 NLS-Rluc and pTargetB2(0x)-LexA-Rluc) (Fig. 7b). As for the 2 day delay of pCMV-int 14 transfection, the targeted integration efficiency of pTargetB2(4 18x)-LexA-Rluc was remarkably (up 15 to 26-fold) higher than that of the negative controls (Fig. 7c). Unexpectedly, the targeted integration 16 efficiencies of pTargetB2(66x)-LexA-Rluc were lower than that of pTargetB2(4 18x)-LexA-Rluc under both transfection conditions.

\section{Confirmation of targeted integration by sequencing analysis}

To confirm integration into the attP close to the LexA operator sequence, a plasmid rescue method was adopted. First, Hela-attPlex4R cells were transfected with pTargetB2(4x)-LexA-Rluc and, 2 days later, with pCMV-int, and then selected by treatment with blasticidin. Secondly, genomic DNA was extracted from the cells, digested with restriction enzymes, ligated, and used for E. coli transformation. As pTargetB2(4x)-LexA-Rluc contains a blasticidin-resistance gene and E. coli replication origin, fragments of genomic DNA containing an insertion of pTargetB2(4x)-LexA-Rluc can be replicated in the presence of blasticidin. Of the obtained 2 clones of phiC31 integrase-mediated integration products, one is the targeted integration product (Table 1). Surprisingly, the other is 
integrated into pseudo-attP in pCMV-int.

\section{Discussion}

The present study was designed to enhance the integration site-specificity of phiC31 integrase-based vectors by tethering the donor vector to chromosomes with a target sequence-specific dual DNA-binding protein. Although the strategic concept has already been applied to Sleeping Beauty-based vectors (Ivics et al., 2007), we made some further improvements to adapt the method for phiC31 integrase-mediated integration. First, we incorporated an expression cassette for a dual DNA-binding protein in the donor vectors, instead of constructing donor vectors and the DNA-binding protein expression vectors separately. This guarantees the expression of the DNA-binding protein in cells transfected with the donor vectors. Secondly, we inserted multiple $(4 \sim 66 \mathrm{x})$ binding sequences into the donor vectors to increase the probability of binding the DNA-binding protein to the donor vectors. Thirdly, we transfected cells with the donor vectors in advance of transfection with the helper vector in order to ensure expression of the DNA-binding protein and tethering of the donor vectors at the target sequence of chromosomes prior to phiC31 integrase expression.

Under the modified conditions, the donor vectors containing dual DNA-binding protein provided a higher integration site-specificity than their negative controls in both the inter-plasmid integration assay (Fig. 4b) and chromosomal integration assay (Fig. 7c). In previous studies using sequence-specific DNA-binding motifs, the fold increases of targeted chromosomal integration ranged from 2.0 to 9.9 (Ivics et al., 2007, Kettlun et al., 2011, Owens et al., 2012, Tan et al., 2006, Voigt et al., 2012). Even though our data cannot simply be compared with the previous data because of the differences in evaluation and analysis methods, a 26 -fold increase in targeted chromosomal integration (Fig. 7c) would be considerably large. However, the percentage of the donor vector integrated into native $a t t P$ (not pseudo- $a t t P)$ neighboring the target sequence was not necessarily high $(0.38 \%$ of tonal donor vectors; Fig. 7b). One reason for the low rate of targeted integration might be associated with the context of attP-surrounding sequence (Calos, 2006). The inter-plasmid integration assay showed 
that the integration efficiency of pTargetB-NLS-Rluc into pAttP-lex4R was lowest among all recipient vectors (Fig. 4a). This suggests that the context of pAttP-lex4R-derived surrounding sequences might not be suitable for phiC31 integrase-mediated integration. Therefore, if we select attP or pseudo-attP sequences with more suitable surrounding contexts, the targeted integration percentage may increase. In Hela-attPlex4R that was used for chromosomal integration assay, multiple target attP sequences were inserted into the genome (15 copies per haploid genome). However, all the target attP sites might not necessarily be available for transgene expression due to their surrounding chromosomal contexts and epigenetic modifications. Selection of blasticidin-resistant cells could lead to underestimation of the degree of site-specific chromosomal integration of pTargetB2-LexA-Rluc, since it rules out integration to the target attP sites that do not allow the expression of the resistance marker. Taking together with the existence of pseudo-attP sites in some regions of the genome such as heterochromatin, it would be difficult to determine an exact efficiency count of targeted chromosomal integration. Thus, it should be noted that the present assay method of targeted integration simply allows relative comparison among the vector systems.

In the chromosomal integration assay, delayed transfection of the helper vector after that of the donor vector transfections increased the difference between the pTargetB2( $4 \sim 18 \mathrm{x})$-LexA-Rluc) and the negative controls (pTargetB2-NLS-Rluc and pTargetB2(0x)-LexA-Rluc) (Fig. 7b, c). These results suggest that the time-lag transfection provides an opportunity for the DNA-binding proteins to tether the donor vectors to the target sequences before phiC31 integrase-mediated integration. In addition to time-lag transfection, the use of chemical-regulatable gene expression systems is another option for producing an expression time-lag (Sharma et al., 2008 and Yen et al., 2004).

Because of the simplicity of evaluation, the LexA operator sequence was selected as a target sequence of the DNA-binding protein by inserting it into chromosomes exogenously. However, when this targeted integration strategy is intended for practical applications such as gene therapies, the target sequences should be selected from native genomic sequences. To achieve safer chromosomal integration, pseudo-attP sequences in genomic safe harbors (Sadelain et al., 2012) should be selected as integration sites, and a specific DNA-binding motif should be customized so that it binds near the 
1 pseudo-attP sequences. In addition, it has been established that pseudo-attP sequences that can be

2 recognized as substrates for phiC31 integrase exhibit certain variations in chromosomal context among

3 cell types (Calos, 2006). Selection of different target sequences might thus be required depending on

4 the cell types.

The present targeted integration approach is theoretically applicable to other integrative vector systems. Ivics et al. have shown that an LexA-SAFbox DNA-binding protein did not inhibit

7 Sleeping Beauty-mediated integration (Ivics et al., 2007), and during the preparation of this manuscript,

8 Owens et al. reported targeted piggyBac integration by tethering of the donor vectors (Owens et al.,

9 2013). As experienced with problem associated with the attB site (Fig. 6), the design of donor vectors

10 appears to be important in targeted integration. As long as the vectors are carefully designed, the

11 present targeted integration approach would be useful for other integrative vector systems, including

$12 \quad$ Sleeping Beauty and piggyBac.

13 In conclusion, we demonstrated by using phiC31 integrase-based integration systems that a

14 multi-functional donor vector which expresses a sequence-specific dual DNA-binding protein that

15 tethers itself to the target sequence and which has multiple sequences for binding of the protein works

16 cooperatively with a helper vector when cells are subjected to both vector transfections separated by

17 an appropriate time lag. We believe that the present study provides important information toward the

18 achievement of site-specific transgene integration, and paves the way for more reliable gene therapies

19 and genetic studies.

21 Acknowledgements

We thank Hajime Mori (Kyoto Institute of Technology, Kyoto, Japan) and Takahiro

24 Kusakabe (Kyushu University, Fukuoka, Japan) for p3E1.2 (a plasmid DNA containing the piggyBac

25 original fragment) and pZerofd31attB3xP3EGFP (a plasmid DNA containing attB sequence),

26 respectively. This work was supported by the Japan Society for the Promotion of Science (KAKENHI

27 Grant Number 11J01309), Grants-in-Aid for Scientific Research (Number 23659284 and 25670259) 
1 from the Ministry of Education, Culture, Sports, and Science, and the PRESTO program (Number

2 3424) of the Japan Science and Technology Agency. None of the authors have any conflicts of interest

3 associated with this study.

\section{References}

Allen, B.G., Weeks, D.L., 2005. Transgenic Xenopus laevis embryos can be generated using ФC31 integrase. Nat. Methods 2, 975-979.

Ammar, I., Gogol-Döring, A., Miskey, C., Chen, W., Cathomen, T., Izsvák, Z., Ivics, Z., 2012. Retargeting transposon insertions by the adeno-associated virus Rep protein. Nucleic Acids Res. 40, 6693-712.

Calos, M.P., 2006. The ФC31 Integrase System for Gene Therapy. Curr. Gene Ther. 6, 633-645.

Carlson, C.M., Frandsen, J.L., Kirchhof, N., Mcivor, R.S., Largaespada, D.A., 2005. Somatic integration of an oncogene-harboring Sleeping Beauty transposon models liver tumor development in the mouse. Proc. Natl. Acad. Sci. Unites States Am. 102, 17059-17064.

Carroll, D., Morton, J.J., Beumer, K.J., Segal, D.J., 2006. Design, construction and in vitro testing of zinc finger nucleases. Nat. Protoc. 1, 1329-1341.

Cermak, T., Doyle, E.L., Christian, M., et al., 2011. Efficient design and assembly of custom TALEN and other TAL effector-based constructs for DNA targeting. Nucleic Acids Res. 39, e82.

Chalberg, T.W., Portlock, J.L., Olivares, E.C., et al., 2006. Integration specificity of phage phiC31 integrase in the human genome. J. Mol. Biol. 357, 28-48.

Cong, L., Ran, F.A., Cox, D., et al., 2013. Multiplex genome engineering using CRISPR/Cas systems. Science 339, 819-23.

Gersbach, C.A., Gaj, T., Gordley, R.M., Barbas, C.F., 2010. Directed evolution of recombinase specificity by split gene reassembly. Nucleic Acids Res. 38, 4198-206.

Gordley, R.M., Gersbach, C.A., Barbas, C.F., 2009. Synthesis of programmable integrases. Proc. Natl. Acad. Sci. U. S. A. 106, 5053-5058. 
1 Goussard, S., Grillot-Courvalin, C., Courvalin, P., 2003. Eukaryotic promoters can direct protein synthesis in Gram-negative bacteria. J. Mol. Microbiol. Biotechnol. 6, 211-218.

Grabundzija, I., Irgang, M., Mátés, L., et al., 2010. Comparative analysis of transposable element vector systems in human cells. Mol. Ther. 18, 1200-9.

Groth, A.C., Fish, M., Nusse, R., Calos, M.P., 2004. Construction of Transgenic Drosophila by Using the Site-Specific Integrase From Phage ФС31. Genetics 166, 1775-1782.

Groth, A.C., Olivares, E.C., Thyagarajan, B., Calos, M.P., 2000. A phage integrase directs efficient site-specific integration in human cells. Proc. Natl. Acad. Sci. U. S. A. 97, 5995-6000.

Hacein-Bey-Abina, S., Von Kalle, C., Schmidt, M., et al., 2003. LMO2-associated clonal T cell proliferation in two patients after gene therapy for SCID-X1. Science 302, 415-419.

Ivics, Z., Katzer, A., Stüwe, E.E., Fiedler, D., Knespel, S., Izsvák, Z., 2007. Targeted Sleeping Beauty transposition in human cells. Mol. Ther. 15, 1137-1144.

Keravala, A., Lee, S., Thyagarajan, B., Olivares, E.C., Gabrovsky, V.E., Woodard, L.E., Calos, M.P., 2009. Mutational Derivatives of PhiC31 Integrase With Increased Efficiency and Specificity. Mol. Ther. 17, 112-120.

Kettlun, C., Galvan, D.L., George, A.L., Kaja, A., Wilson, M.H., 2011. Manipulating piggyBac transposon chromosomal integration site selection in human cells. Mol. Ther. 19, 1636-44.

Lacoste, A., Berenshteyn, F., Brivanlou, A.H., 2009. An efficient and reversible transposable system for gene delivery and lineage-specific differentiation in human embryonic stem cells. Cell Stem Cell 5, 332-42.

Leighton, P. a, van de Lavoir, M.-C., Diamond, J.H., Xia, C., Etches, R.J., 2008. Genetic modification of primordial germ cells by gene trapping, gene targeting, and phiC31 integrase. Mol. Reprod. Dev. 75, 1163-75.

Mali, P., Yang, L., Esvelt, K.M., Aach, J., Guell, M., DiCarlo, J.E., Norville, J.E., Church, G.M., 2013. RNA-guided human genome engineering via Cas9. Science 339, 823-6.

Mandell, J.G., Barbas, C.F., 2006. Zinc Finger Tools: custom DNA-binding domains for transcription factors and nucleases. Nucleic Acids Res. 34, W516-W523. 
Maragathavally, K.J., Kaminski, J.M., Coates, C.J., 2006. Chimeric Mos1 and piggyBac transposases result in site-directed integration. FASEB J. 20, 1880-2.

Miller, J.C., Tan, S., Qiao, G., et al., 2011. A TALE nuclease architecture for efficient genome editing. Nat. Biotechnol. 29, 143-8.

Nakanishi, H., Higuchi, Y., Kawakami, S., Yamashita, F., Hashida M., 2010. piggyBac Transposonmediated Long-term Gene Expression in Mice. Mol. Ther. 18, 707-714.

Nakanishi, H., Higuchi, Y., Kawakami, S., Yamashita, F., Hashida, M., 2011. Comparison of piggyBac transposition efficiency between linear and circular donor vectors in mammalian cells. J. Biotechnol. 154, 205-208.

Olivares, E.C., Hollis, R.P., Chalberg, T.W., Meuse, L., Kay, M.A., Calos, M.P., 2002. Site-specific genomic integration produces therapeutic Factor IX levels in mice. Nat. Biotechnol. 20, $1124-1128$.

Owens, J.B., Mauro, D., Stoytchev, I., Bhakta, M.S., Kim, M.-S., Segal, D.J., Moisyadi, S., 2013. Transcription activator like effector (TALE)-directed piggyBac transposition in human cells. Nucleic Acids Res. Epub ahead.

Owens, J.B., Urschitz, J., Stoytchev, I., et al., 2012. Chimeric piggyBac transposases for genomic targeting in human cells. Nucleic Acids Res. 40, 6978-6991.

Robertson, G., Garrick, D., Wu, W., Kearns, M., Martin, D., Whitelaw, E., 1995. Position-dependent variegation of globin transgene expression in mice. Proc. Natl. Acad. Sci. U. S. A. 92, 53715.

Sadelain, M., Papapetrou, E.P., Bushman, F.D., 2012. Safe harbours for the integration of new DNA in the human genome. Nat. Rev. Cancer 12, 51-58.

Sharma, N., Moldt, B., Dalsgaard, T., Jensen, T.G., Mikkelsen, J.G., 2008. Regulated gene insertion by steroid-induced PhiC31 integrase. Nucleic Acids Res. 36, e67.

Shinohara, E.T., Kaminski, J.M., Segal, D.J., et al., 2007. Active integration: new strategies for transgenesis. Transgenic Res. 16, 333-9.

Takahashi, K., Yamanaka, S., 2006. Induction of pluripotent stem cells from mouse embryonic and 
adult fibroblast cultures by defined factors. Cell 126, 663-676.

Tan, W., Dong, Z., Wilkinson, T.A., Barbas, C.F.I., Chow, S.A., 2006. Human Immunodeficiency Virus Type 1 Incorporated with Fusion Proteins Consisting of Integrase and the Designed Polydactyl Zinc Finger Protein E2C Can Bias Integration of Viral DNA into a Predetermined Chromosomal Region in Human Cells. J. Virol. 80, 1939-1948.

Tan, W., Zhu, K., Segal, D.J., Barbas, C.F.I., Chow, S.A., 2004. Fusion Proteins Consisting of Human Immunodeficiency Virus Type 1 Integrase and the Designed Polydactyl Zinc Finger Protein E2C Direct Integration of Viral DNA into Specific Sites. J. Virol. 78, 1301-1313.

Thomason, L.C., Calendar, R., Ow, D.W., 2001. Gene insertion and replacement in Schizosaccharomyces pombe mediated by the Streptomyces bacteriophage $\varphi \mathrm{C} 31$ sitespecific recombination system. Mol. Genet. Genomics 265, 1031-1038.

Thorpe, H.M., Smith, M.C., 1998. In vitro site-specific integration of bacteriophage DNA catalyzed by a recombinase of the resolvase/invertase family. Proc. Natl. Acad. Sci. U. S. A. 95, 550510.

Thyagarajan, B., Olivares, E.C., Hollis, R.P., Ginsburg, D.S., Calos, M.P., 2001. Site-Specific Genomic Integration in Mammalian Cells Mediated by Phage ФC31 Integrase. Mol. Cell. Biol. 21, 3926-3934.

Tomita, M., Munetsuna, H., Sato, T., et al., 2003. Transgenic silkworms produce recombinant human type III procollagen in cocoons. Nat. Biotechnol. 21, 52-6.

Urnov, F.D., Miller, J.C., Lee, Y.-L., et al., 2005. Highly efficient endogenous human gene correction using designed zinc-finger nucleases. Nature 435, 646-651.

Vink, C.A., Gaspar, H.B., Gabriel, R., Schmidt, M., Mcivor, R.S., Thrasher, A.J., Qasim, W., 2009. Sleeping Beauty Transposition From Nonintegrating Lentivirus. Mol. Ther. 17, 1197-1204.

Voigt, K., Gogol-Döring, A., Miskey, C., Chen, W., Cathomen, T., Izsvák, Z., Ivics, Z., 2012. Retargeting Sleeping Beauty Transposon Insertions by Engineered Zinc Finger DNAbinding Domains. Mol. Ther. 20, 1852-1862.

Watanabe, S., Nakamura, S., Sakurai, T., Akasaka, K., Sato, M., 2011. Improvement of a phiC31 
2

3

integrase-based gene delivery system that confers high and continuous transgene expression. N. Biotechnol. 28, 312-9.

Wright, D.A., Thibodeau-Beganny, S., Sander, J.D., et al., 2006. Standardized reagents and protocols for engineering zinc finger nucleases by modular assembly. Nat. Protoc. 1, 1637-1652.

Yant, S.R., Huang, Y., Akache, B., Kay, M.A., 2007. Site-directed transposon integration in human cells. Nucleic Acids Res. 35, e50.

Yen, L., Svendsen, J., Lee, J., Gray, J.T., 2004. Exogenous control of mammalian gene expression through modulation of RNA self-cleavage. Nature 431, 471-476.

Zhang, F., Cong, L., Lodato, S., Kosuri, S., Church, G.M., Arlotta, P., 2011. Efficient construction of sequence-specific TAL effectors for modulating mammalian transcription. Nat. Biotechnol. $29,149-154$. 
1 Fig. 1 Schematic diagram of targeted integration using a sequence-specific DNA-binding

2 protein and phiC31 integrase

3 Gal4 and LexA DNA-binding domains of the Gal4-NLS-LexA DNA-binding protein bind to UAS and

4 the LexA operator (the target sequence), respectively. Then, phiC31 integrase integrates the donor

$5 \quad$ vector by recombining the $a t t B$ and $a t t P$ sequences. Because the donor vector was tethered to the target

6 sequence, its integration sites are restricted to the vicinity of the target sequence.

\section{Fig. 2 Vectors for targeted integration}

9 Vector construction. $A \mathrm{mp}^{\mathrm{R}}$, ampicillin-resistance gene; $a t t B$, attB sequence for phiC31 integrase10 mediated recombination; $a t t P, a t t P$ sequence for phiC31integrase-mediated recombination; Blast ${ }^{\mathrm{R}}$, 11 blasticidin-resistance gene; CMV, cytomegalovirus promoter; EM7, bacterial EM7 promoter; Ga14,

12 Gal4 DNA-binding domain; hEF1, human elongation factor $1 \alpha$ promoter; LexA, LexA DNA-binding 13 domain; NLS, nuclear localization signal; Rluc, Renilla luciferase gene; $\mathrm{Neo}^{\mathrm{R}}$, neomycin-resistance 14 gene; SV40, simian virus 40 promoter; ori, E. coli origin of replication; $4 \times \mathrm{UAS}$, four copies of 15 upstream activating sequences; Kan $/ \mathrm{Neo}^{\mathrm{R}}$, kanamycin/neomycin-resistance gene; LexA operator, 16 LexA operator sequence; SV40/BP, simian virus 40 promoter/Bacterial promoter. Each recipient vector (pAttP-lex1 4F and 1 4R) has one copy of attP at 6.5-6.6 (1F, 1R), 1.7-1.8 (2F, 2R), 0.6-0.7

18 (3F, 3R), or 0.2-0.3 (4F, 4R) kb upstream, respectively, from LexA operator start point as indicated orientations.

Fig. 3 Effect of DNA-binding proteins on phiC31 integrase-mediated sustained gene expression HEK293-lexluc cells $\left(2 \times 10^{5}\right.$ cells/well) were transfected with $500 \mathrm{ng}$ of the indicated donor vector and 500 ng pCMV-int or pcDNA3.1(+). The bars show the results for pAttB-UAS-Rluc and

24 pcDNA3.1(+) (white), pAttB-UAS-Rluc and pCMV-int (hatched), pTargetB-NLS-Rluc and pcDNA3.1(+) (dotted), pTargetB-NLS-Rluc and pCMV-int (black), respectively. Renilla luciferase

26 activities were measured at the indicated time points after transfection. Each value represents the mean $27+\mathrm{SD}(\mathrm{n}=3)$. 
2 Fig. 4 Effect of the DNA-binding protein on the integration site selection of phiC31 integrase in 3 inter-plasmid integration

4 Hela cells were transfected with pAttP-lex1F $\sim 4 \mathrm{R}(125 \mathrm{ng}$ each), $1 \mu \mathrm{g}$ pSV40-int $(1 \mu \mathrm{g})$, and

5 pTargetB-LexA-Rluc or pTargetB-NLS-Rluc $(1 \mu \mathrm{g})$. DNA extracted from Hela cells was subjected to

6 E. coli transformation. Recombination products amplified in E. coli were analyzed by restriction

7 digestion and agarose gel electrophoresis. Figure 4(a) shows the percentages of each inter-plasmid

8 integration product per total inter-plasmid integration products. Open rectangles indicate inter-plasmid

9 integration products of pTargetB-LexA-Rluc and each recipient vector. Closed squares indicate inter-

10 plasmid integration products of pTargetB-NLS-Rluc and each recipient vector. Figure 4(b) represents

11 the ratios of pTargetB-LexA-Rluc to pTargetB-NLS-Rluc in percentages of each inter-plasmid 12 integration product. The abscissa indicates the distance between the LexA operator and attP sequences

13 in each recipient vector.

Fig. 5 Establishment of a stable cell line containing LexA operator and attP sequences in chromosomes

17 To analyze integration site-specificity in a chromosomal context, LexA operator and attP sequences were integrated into the chromosomes of Hela cells by piggyBac transposase-mediated integration. $A m p^{\mathrm{R}}$, ampicillin-resistance gene; $a t t P, a t t P$ sequence for phiC31 integrase-mediated recombination; $\mathrm{Kan} / \mathrm{Neo}^{\mathrm{R}}$, kanamycin/neomycin-resistance gene; IR, piggyBac inverted repeat sequence for piggyBac transposase-mediated integration; LexA operator, LexA operator sequence; SV40/BP, simian virus 40 22 promoter/bacterial promoter.

Fig. 6 Colony-forming efficiency of pTargetB-NLS-Rluc and pTargetB2-NLS-Rluc

Figure 6(a) shows the vector construction of pTargetB2-NLS-Rluc and pTargetB2(0 66x)-LexARluc. $a t t B, a t t B$ sequence for phiC31 integrase-mediated recombination; Blast ${ }^{\mathrm{R}}$, blasticidin-resistance gene; CMV, cytomegalovirus promoter; Ga14, Gal4 DNA-binding domain; hEF1, human elongation 
1 factor $1 \alpha$ promoter; LexA, LexA DNA-binding domain; NLS, nuclear localization signal; Rluc,

2 Renilla luciferase gene; SV40/EM7, simian virus 40 promoter/bacterial EM7 promoter; ori, E. coli

3 origin of replication; 0 66xUAS, 0 to 66 copies of upstream activating sequences. Figure 6(b) shows

4 the numbers of blasticidin-resistant colonies obtained following transfection with donor and helper

5 vectors. Hela cells were transfected with the indicated donor vector and pcDNA3.1(+) (white bar) or

6 pCMV-int (black bar). The number of colonies was counted by methylene blue staining after 2 weeks'

7 of selection with blasticidin S. Each value represents the mean $+\operatorname{SD}(n=4)$. The data were analyzed

8 by Dunnett's multiple-comparison test. Figure 6(c) shows photographs of colonies stained with

9 methylene blue.

11 Fig. 7 Effect of the DNA-binding protein on the integration site selection of phiC31 integrase in 12 chromosomal integration

13 Figure 7(a) shows the design of primers used to detect targeted integration. The forward and reverse

14 primers are designed to anneal the nearby LexA operator sequence in chromosome and attB in the 15 donor vectors, respectively. PCR amplified DNA only when targeted integration products are 16 templates. Figure 7(b) and (c) represent percentages of the donor vector integrated into target attP per 17 total donor vector remaining in the cells at 21 days after transfection. The helper vector (pCMV-int) 18 was transfected 1 (b) or 2 (c) days after donor vector transfection. Each value represents the mean + $19 \operatorname{SD}(\mathrm{n}=3)$. 
Table 1

Sequence and locations of phiC31 integrase-mediated integration of pTargetB2(4x)-LexARluc

\begin{tabular}{|ll|}
\hline \multicolumn{1}{|c|}{ Sequences } & \multicolumn{1}{c|}{ Locations } \\
\hline CGCGCCCGGGGAGCCCAATGAATCGGCCAACGCGCGGG & $\begin{array}{l}320 \text { bp upstream of } \\
\text { GAGAGGCGGTTTGCGTATTGGGC }\end{array}$ \\
& pMV promoter in \\
& pCMV-int \\
\hline GGCGCCCGGGGAGCCAAAGGTTACCCCAGTTGGGGCAG & attP located 219 bp \\
TTGGCATTAAATGTC & from LexA operator \\
\hline
\end{tabular}

Columns 1 and 2 show sequences of attR and flanked sequences and locations of integration sites. Sequences with underlines refers pTargetB2(4x)-LexA-Rluc derived sequences. 


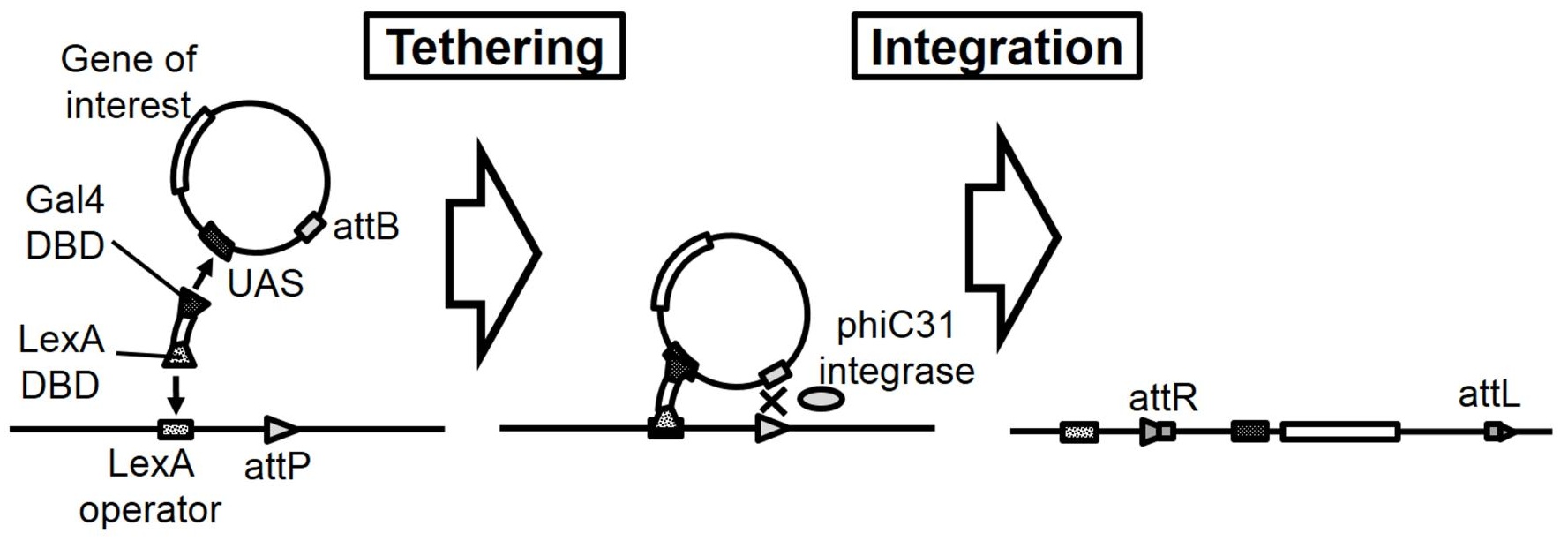



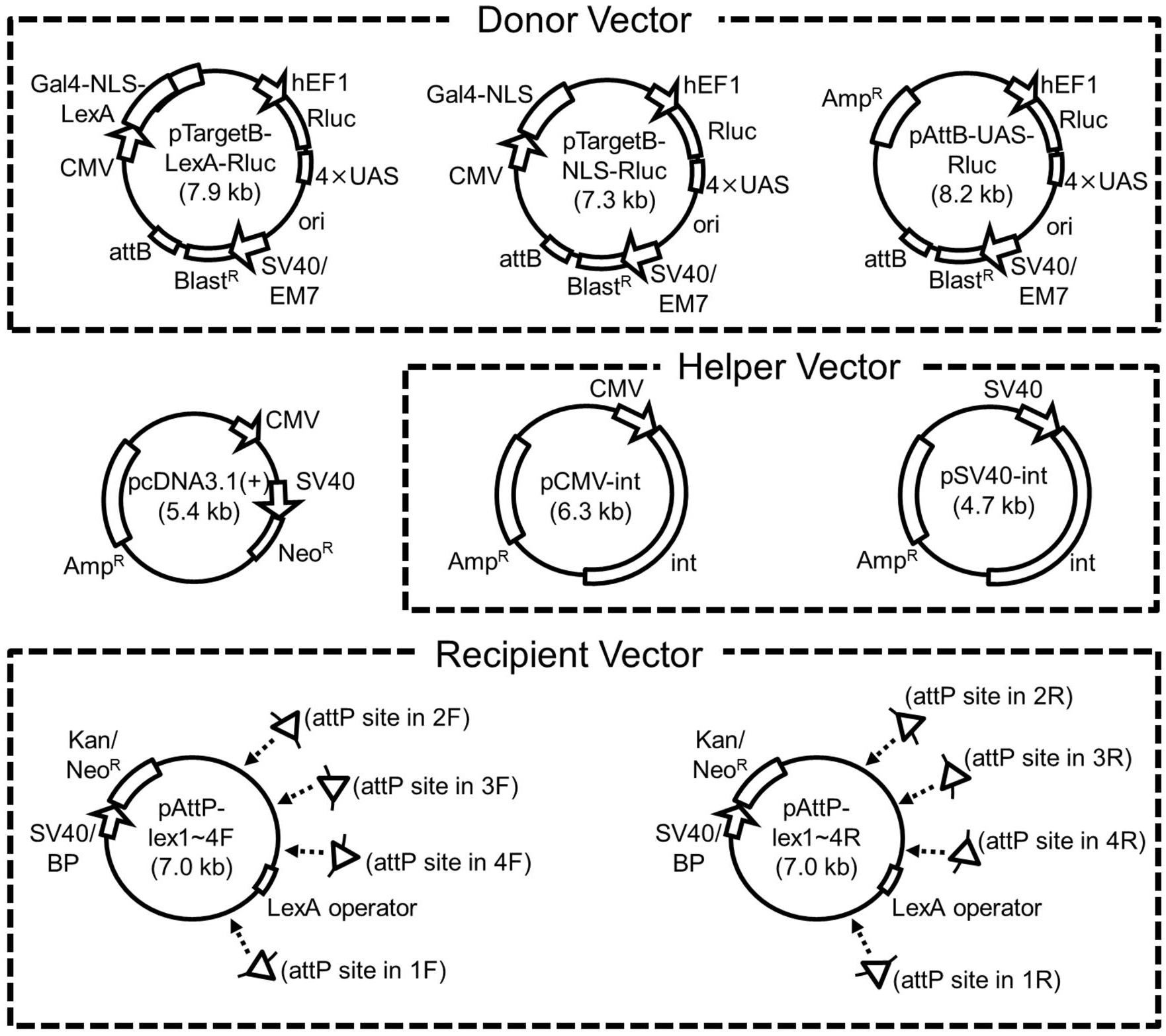


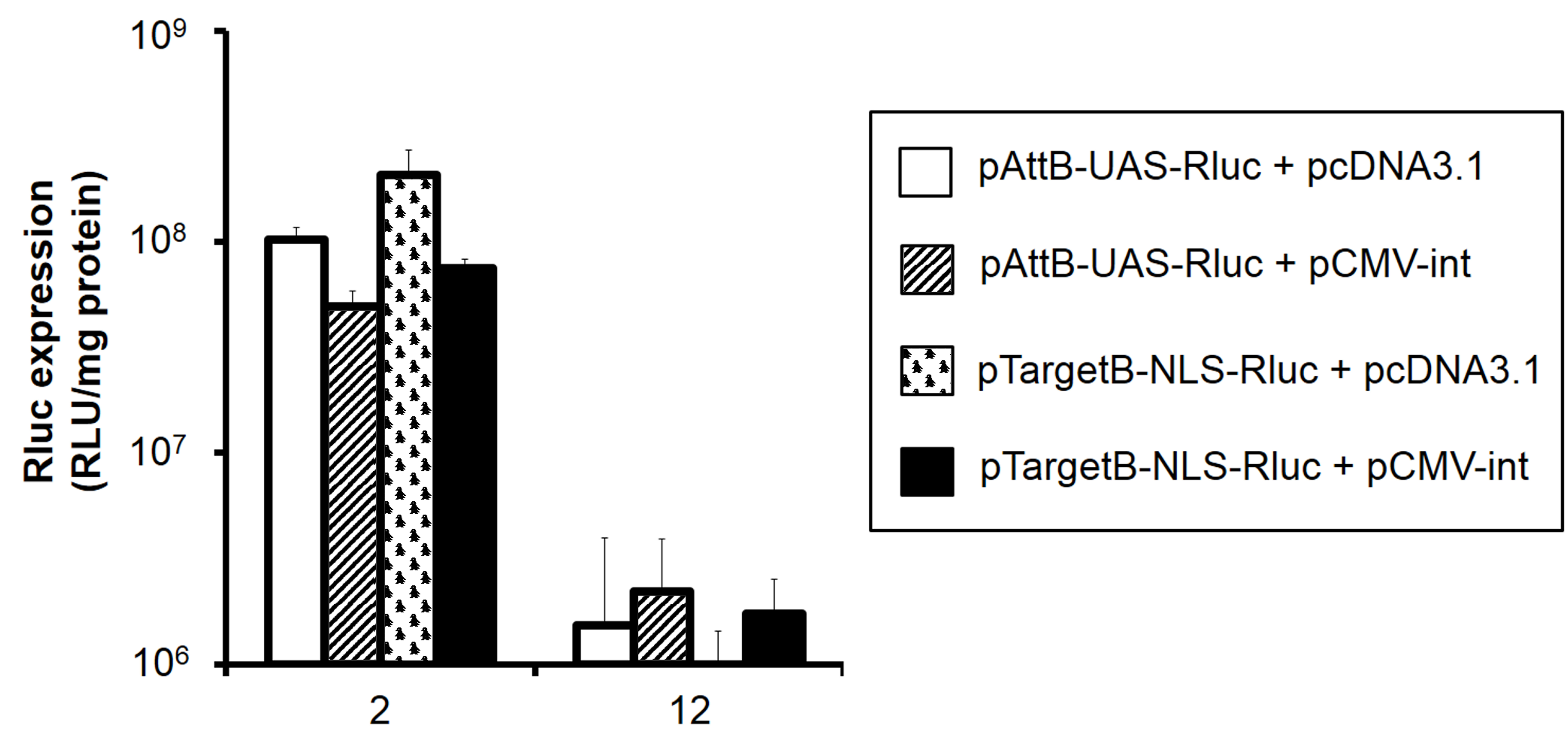

Days after transfection 


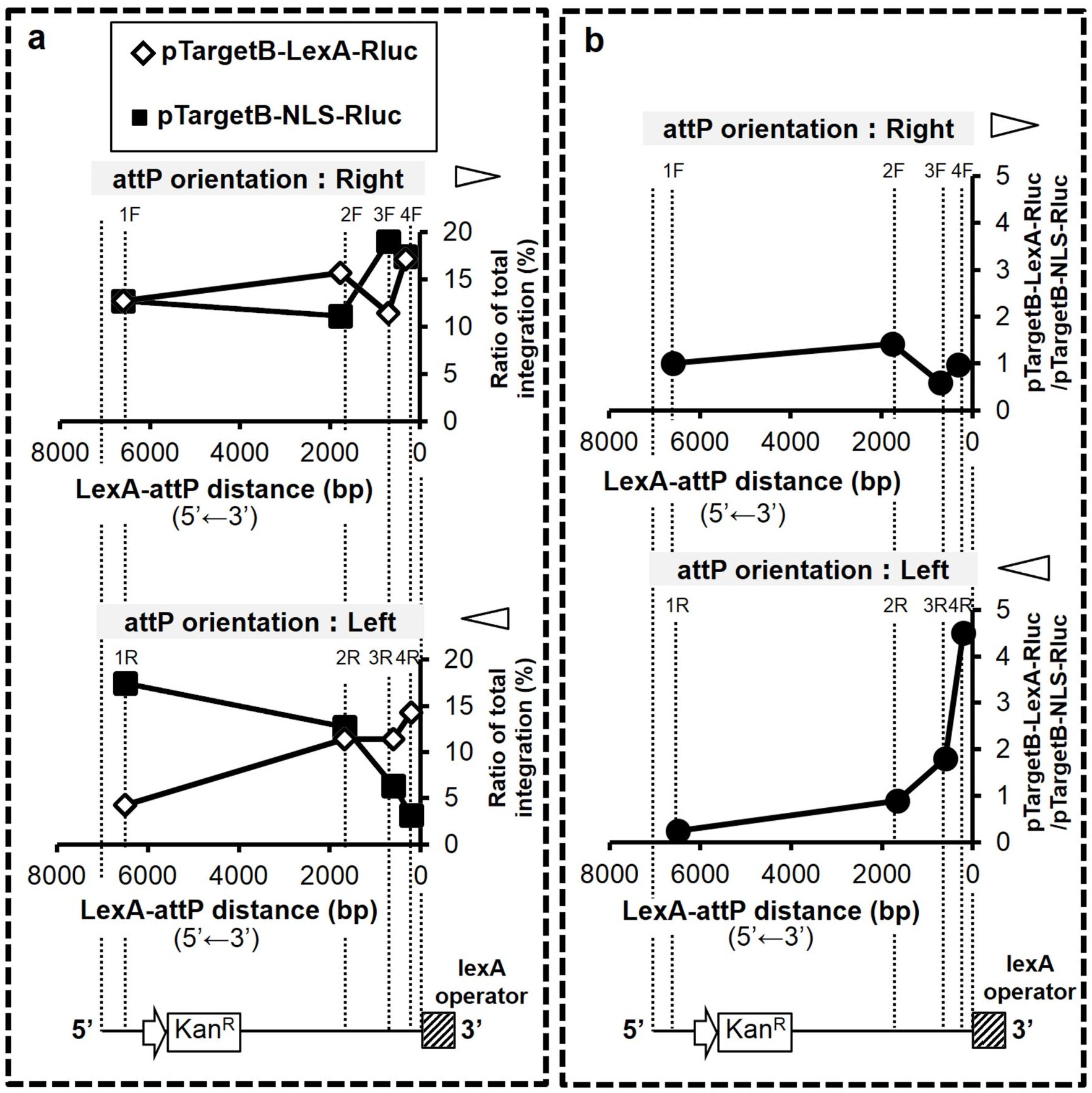




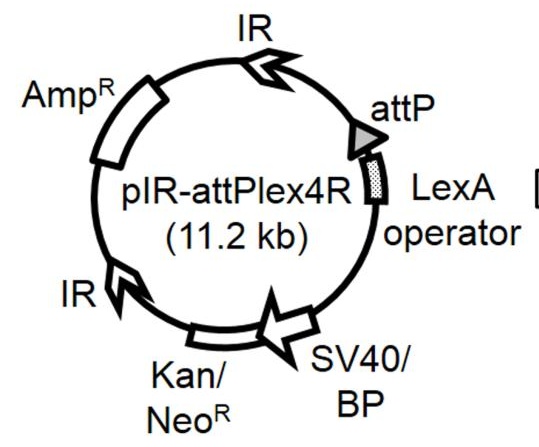

Transposase-mediated integration of LexA operator and att $P$ sequences

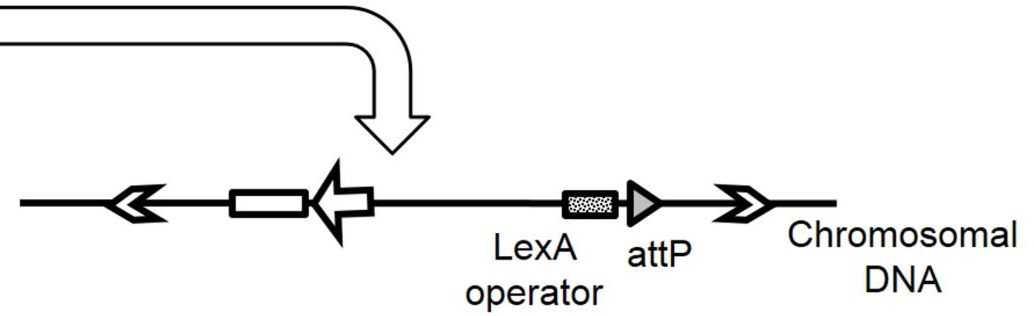



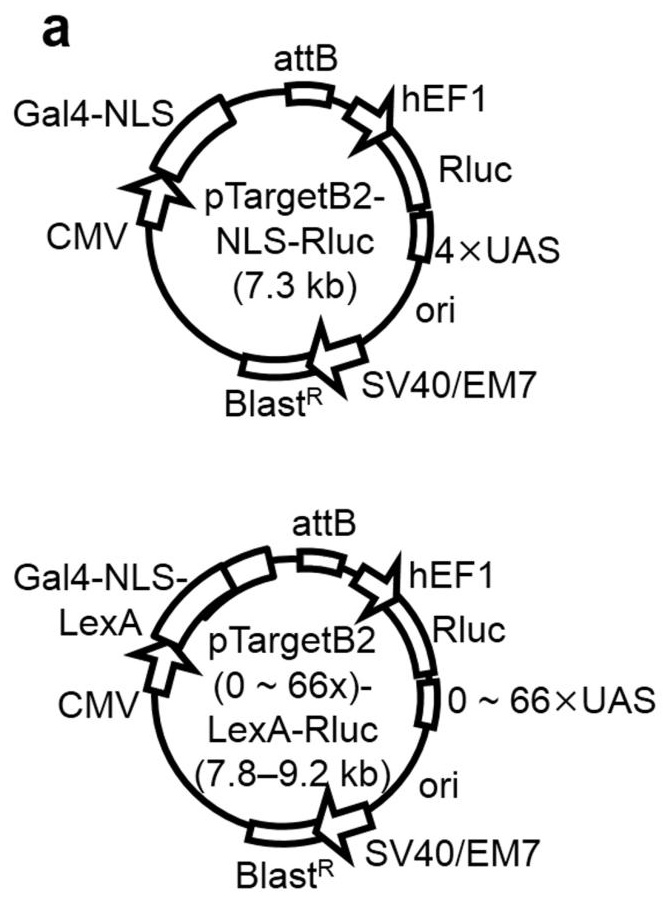

b

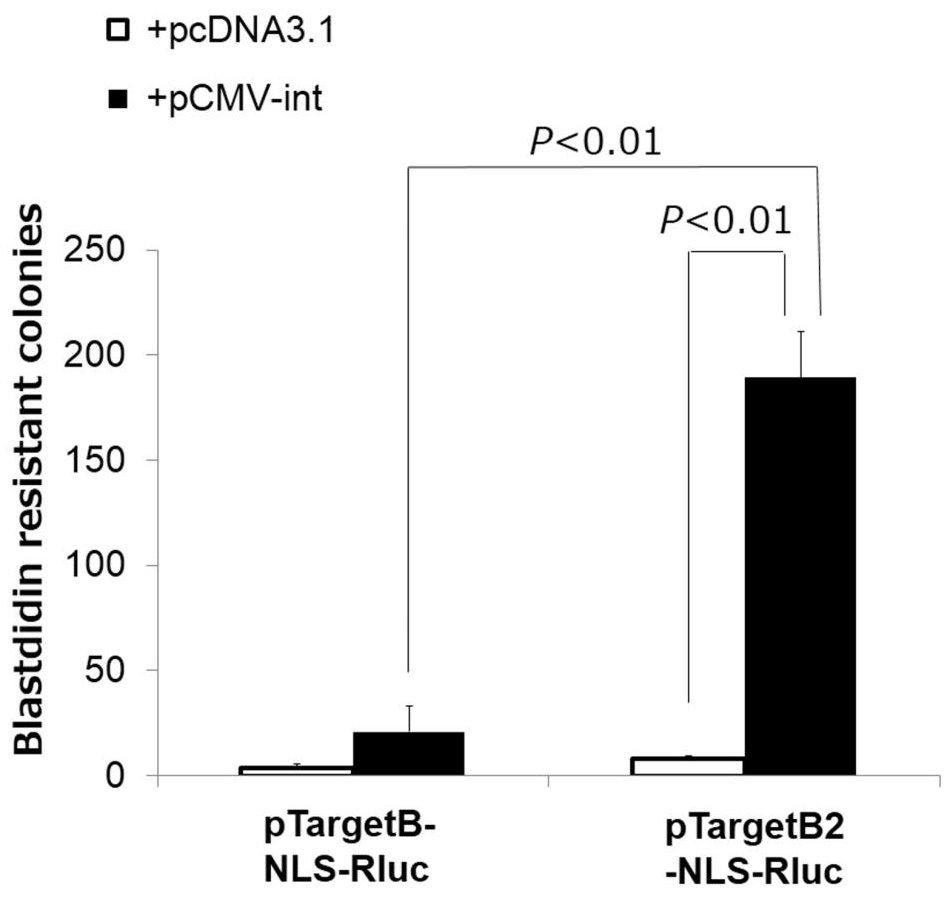

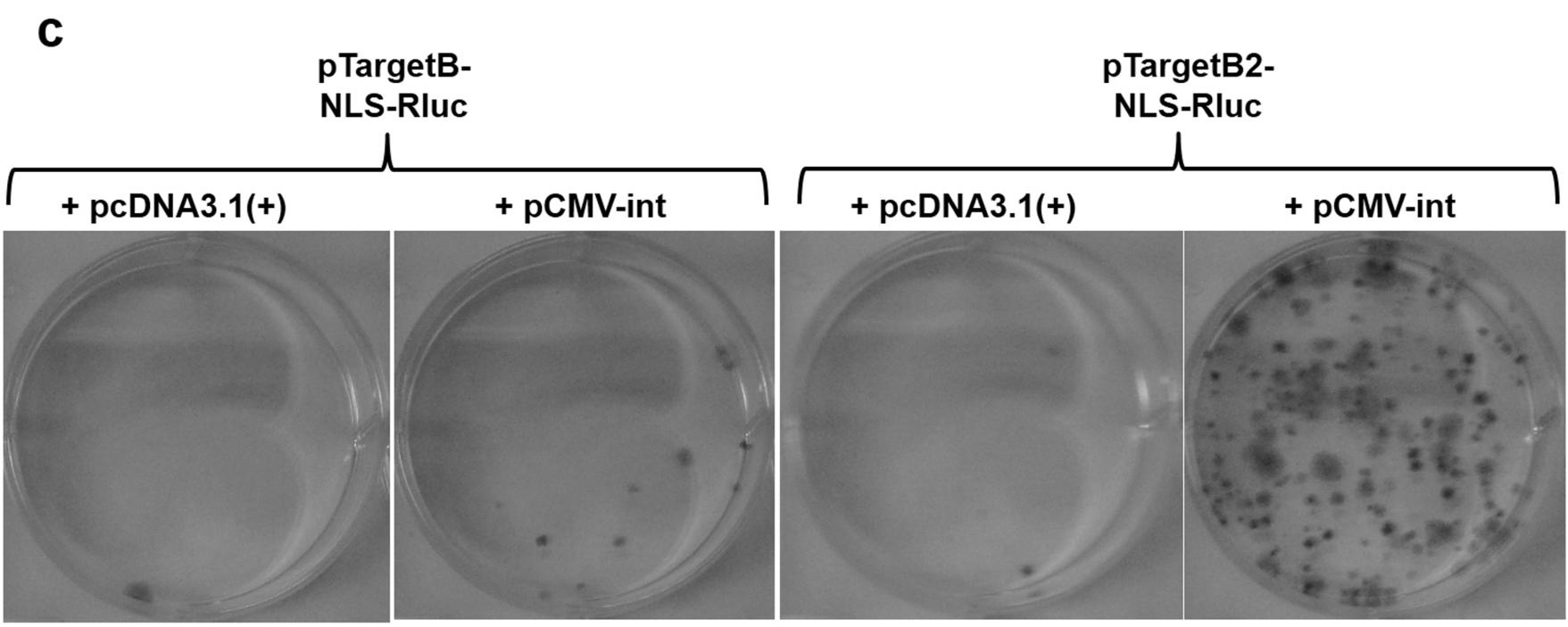


a

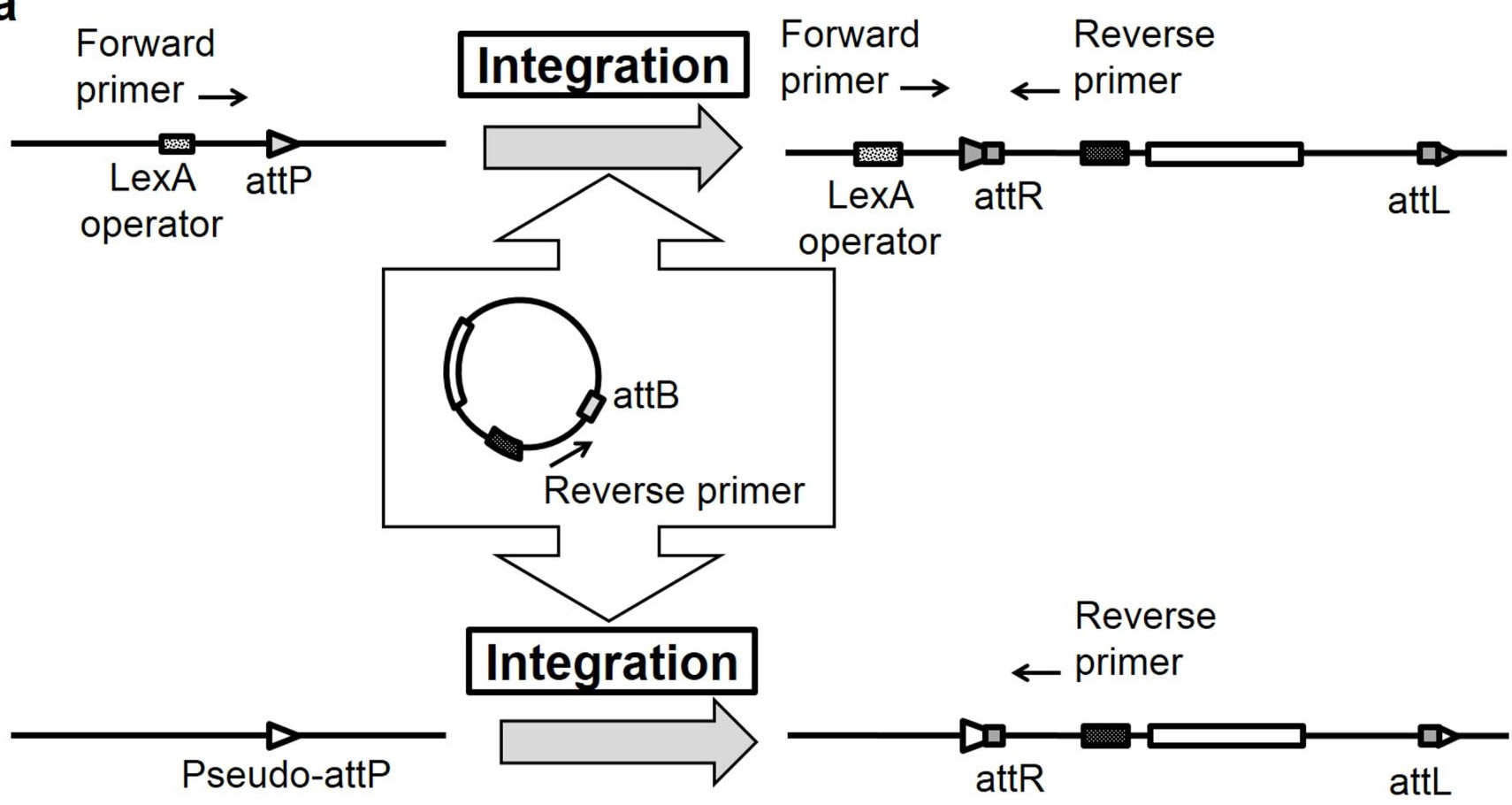

b

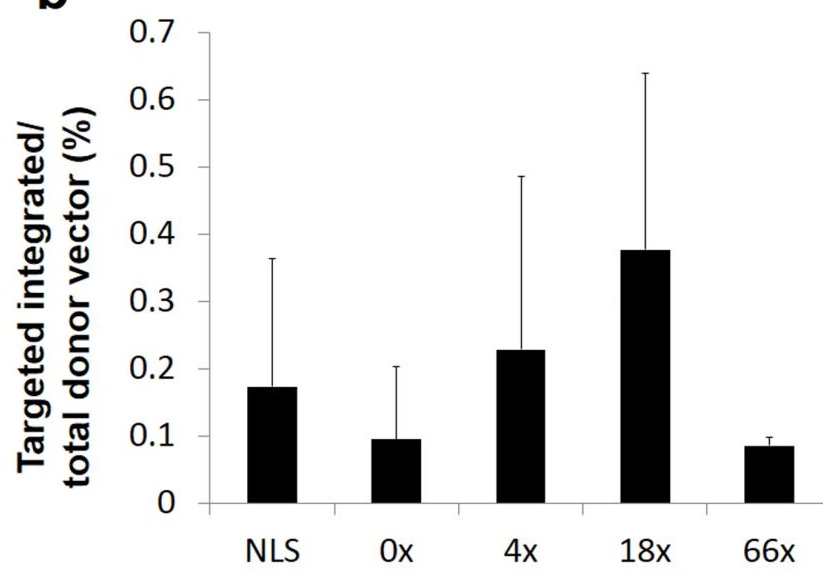

C

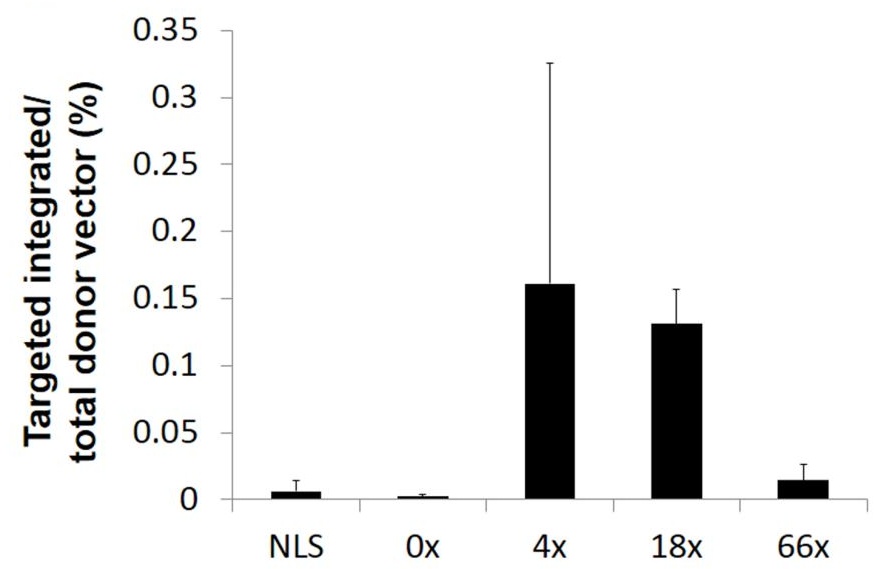

